\title{
In vivo uptake of inhaled particles by airway phagocytes is enhanced in patients with mild asthma compared with normal volunteers
}

\author{
J C Lay, ${ }^{1,2}$ N E Alexis, ${ }^{1,2}$ K L Zeman, ${ }^{1}$ D B Peden, ${ }^{1,23}$ W D Bennett ${ }^{1,3}$
}

\begin{abstract}
- Detailed descriptions of the methods used in this study are published online only at http:// thorax.bmj.com/content/vol64/ issue 4

${ }^{1}$ Center for Environmental Medicine, Asthma and Lung Biology, University of North Carolina School of Medicine, Chapel Hill, North Carolina, USA;

${ }^{2}$ Department of Pediatrics, University of North Carolina School of Medicine, Chapel Hill, North Carolina, USA;

${ }^{3}$ Department of Medicine, University of North Carolina School of Medicine, Chapel Hill, North Carolina, USA
\end{abstract}

Correspondence to: Dr J C Lay, Center for Environmental Medicine, Asthma and Lung Biology, University of North Carolina, Chapel Hill, NC 27599-7310, USA; jcl@med.unc.edu

Received 18 January 2008 Accepted 24 October 2008 Published Online First 26 November 2008

\begin{abstract}
Background: The uptake of inhaled particulate matter by airway phagocytes is an important defence mechanism contributing to the clearance of potentially toxic

substances, including aeroallergens, from the lung. Since airway monocytes and macrophages can also function as antigen presenting cells, their ability to engulf materials deposited on the airway surface is of particular interest in patients with allergic asthma. To determine whether airway mononuclear phagocytes of patients with allergic asthma might have enhanced phagocytic activity, the in vivo uptake of inhaled radiolabelled particles was compared in 10 patients with mild allergic asthma and 8 healthy (non-allergic) individuals.
\end{abstract}

Methods: Phagocyte function was assessed by quantifying the proportion of radioactivity associated with cellular and supernatant fractions of induced sputum $2 \mathrm{~h}$ after inhalation of radiolabelled sulfur colloid particles. All subjects were pretreated with albuterol before sputum induction. A standardised breathing pattern was used to target aerosol deposition in the bronchial airways.

Results: In vivo particle uptake by airway cells was significantly greater in patients with asthma than in healthy volunteers $(57.2 \%(95 \% \mathrm{Cl} 46.5 \%$ to $67.9 \%)$ vs $22.3 \%$ (95\% Cl $4.9 \%$ to $39.6 \%$ ), $\mathrm{p}<0.01$ ), as was in vitro phagocytosis of opsonised zymosan-A bioparticles. There was also a significant correlation $(r=0.85, p<0.01)$ between the percentage of sputum mononuclear phagocytes and the percentage uptake of particles in the patients with asthma but not in the control subjects. Conclusions: In vivo particle uptake by airway macrophages is enhanced in persons with mild asthma. Enhanced uptake and processing of particulate antigens could contribute to the pathogenesis and progression of allergic airways disease and may contribute to the increased risk of disease exacerbation associated with particulate exposure.

Epidemiological studies have shown that particulate matter in the respirable range of $\leqslant 10 \mu \mathrm{m}$ aerodynamic diameter $\left(\mathrm{PM}_{10}\right)$ is associated with a variety of adverse health effects ${ }^{1}$ including respiratory and cardiovascular disease, and have demonstrated a link between particulate air pollution and exacerbations of asthma. ${ }^{2}$ There is also evidence that particles may serve as carriers for biological materials such as endotoxin ${ }^{3}$ and aeroallergens, and that they may also function as adjuvants ${ }^{4}$ by inducing airways inflammation resulting in a "priming" of airway leucocytes involved in airways allergic responses.

Airway mononuclear phagocytes represent one of the first lines of cellular defence against inhaled particulate material including pathogens and allergens, and also probably participate in recall immune responses to either pathogen antigens or allergens. We have previously shown that sputum macrophages from subjects with more severe asthma had impaired phagocytic capacity compared with subjects with less severe asthma and healthy volunteers. ${ }^{5}$ In vitro phagocytosis assays, however, may not accurately reflect the in vivo process which occurs within the airway surface liquid milieu and is influenced by the presence of phagocytosis-modulating factors. Thus, an in vivo methodological approach is needed to assess accurately whether subjects with asthma have constitutively modified particle uptake compared with healthy controls.

We and others have shown that sputum macrophages in healthy individuals rapidly engulf inhaled particles. ${ }^{67}$ Radiolabelled aerosols and induced sputum ${ }^{6}$ were used to examine possible differences in the uptake of inhaled particles by airway phagocytes in patients with mild asthma and healthy volunteers.

\section{METHODS}

Detailed descriptions of the methods used in this study can be found in the online supplement.

\section{Subjects}

Eight healthy non-smoking volunteers aged 19-50 years ( 5 men, 3 women) and 10 subjects with mild atopic asthma aged $22-46$ years ( 2 men, 8 women) were recruited to participate in the study. All subjects had to be able to produce an adequate induced sputum sample (at least $5 \times 10^{5}$ cells) during their screening visit to participate in the study. They had all been free of respiratory tract infections for 6 weeks before beginning the study and had a forced expiratory volume in $1 \mathrm{~s}\left(\mathrm{FEV}_{1}\right)$ of $>80 \%$ of predicted values for a population of similar height, weight, sex, age and race. All the subjects with asthma had physician-diagnosed mild and well controlled asthma and had lung function (percentage predicted $\mathrm{FEV}_{1}$ ) in the normal range with a mean (SEM) percentage predicted $\mathrm{FEV}_{1}$ of $106 \%$ (5\%). With the exception of one subject, all those with asthma had a positive skin test to at least one aeroallergen that included house dust mite antigen, and had a positive methacholine challenge test $\left(\mathrm{PC}_{20} 0.3-10 \mathrm{mg} / \mathrm{ml}\right)$. All the subjects with asthma used an inhaled $\beta$ agonist (albuterol) on an as-needed basis and maintained their allergy medicines (Advair, Singular, Claratin, Allegra, Zyrtec) during the course of the study. 


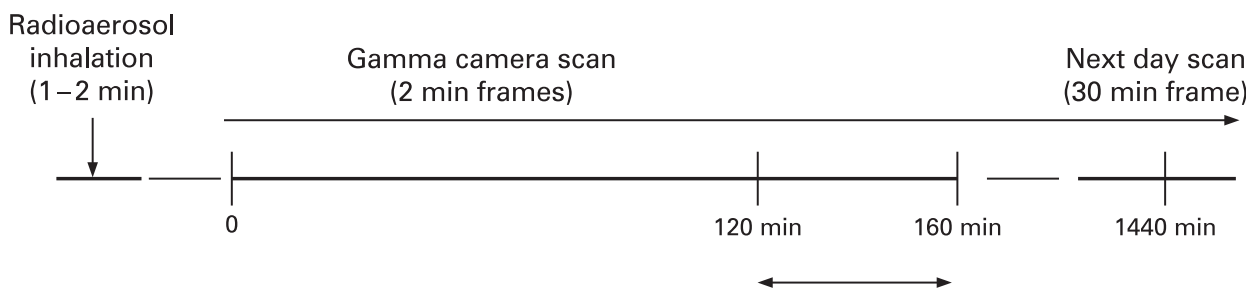

Sputum induction

Figure 1 Experimental design. Dynamic gamma scintigraphy was used to monitor thoracic radiation beginning immediately after inhalation of a radiolabelled particulate aerosol. Sputum induction was performed 120-160 min after inhalation. Additional static scintigraphic images were acquired immediately following sputum induction and again at $24 \mathrm{~h}$ after inhalation.

One asthmatic subject was on an inhaled steroid (Flovent, $100 \mu \mathrm{g}$ twice daily).

\section{Experimental design}

The experimental design is shown in schematic form in fig 1. Subjects first inhaled radiolabelled particles (with deposition targeted to the central bronchial airways), with subsequent monitoring of mucociliary clearance by gamma scintigraphy. We then assessed in vivo uptake of inhaled radiolabelled particles by airway phagocytes recovered from induced sputum at $2 \mathrm{~h}$ following radioaerosol inhalation.

\section{Radioaerosol inhalation and gamma scintigraphy}

Radiolabelling and inhalation of particles and the subsequent gamma scintigraphy have been described previously. ${ }^{9}$ Using a controlled shallow breathing pattern, subjects briefly inhaled $(<2 \mathrm{~min}, \approx 20 \mu \mathrm{Ci}$ total deposited radioactivity) an aerosol of radiolabelled sulfur colloid ( $99 \mathrm{~m} \mathrm{Tc}-\mathrm{SC})$ particles $(0.22 \mu \mathrm{m}$ aerodynamic diameter). Particle retention was monitored using sequential ( $2 \mathrm{~min}$ ) gamma camera scans beginning immediately after inhalation and continuing for $2 \mathrm{~h}$ until sputum induction, with additional scans following sputum induction (40 min later) and at $24 \mathrm{~h}$.

Figure 2 is a posterior scintigraphic image depicting a region of interest drawn over the right lung (large square). The degree of central (C) to peripheral (P) airway particle deposition was calculated as a central to peripheral $(\mathrm{C} / \mathrm{P})$ ratio of ${ }^{99 \mathrm{~m}} \mathrm{Tc}$ activity using counts from the initial 2 min scan following aerosol inhalation and normalised to an ${ }^{133}$ xenon equilibrium scan. ${ }^{8}$ Whole lung particle retention/clearance and the average particle retention was calculated using data only from the right lung to avoid interference of activity from the stomach below the left lung.

\section{Sputum induction and processing}

Methods used for sputum induction (using inhaled hypertonic saline) and processing methods have been described in detail elsewhere. ${ }^{611}$ For safety reasons, patients with asthma must be pretreated with two puffs of albuterol $(180 \mu \mathrm{g})$ before starting hypertonic saline inhalations. To avoid this as a possible confounder, both subjects with asthma and healthy subjects inhaled albuterol before sputum induction. A cell-enriched "select" sample was obtained using thumb forceps to pluck all visible clumps of cells and cell-rich mucus "plugs" from the raw sputum sample. Only the selected sputum was processed further with dithiothrietol (DTT) to remove mucus, since the non-selected secretions have a low proportion of phagocytes and a high proportion (>90\%) of squamous epithelial cells. ${ }^{12}{ }^{13}$

\section{Assessment of in vivo particle uptake}

In vivo uptake of SC particles by airway phagocytes was estimated as the percentage of radioactivity associated with the cell pellet derived from the select sputum samples as previously described. ${ }^{6}$ A correction factor was applied to account for a small proportion of free radiolabelled particles that tended to spin down with the cell pellet following low level centrifugation. ${ }^{6}$

\section{Sputum cell analysis}

Following determination of total and differential cell counts and cell viability, the remaining cells were used for flow cytometric assessment of cell surface phenotypes (mCD14, CD11b/CR3, CD64/Fc $\gamma R I$, CD16/Fc $\gamma R I I I$, CD80/B7.1, CD86/B7.2, HLA-DR) and in vitro phagocytosis assays using a FACSort flow cytometer and CellQuest Pro V.5.2 software (Beckton Dickenson, San Jose, California, USA), as previously described in detail. ${ }^{11}$ Sputum cell populations were identified and gated based on light scatter properties and positive expression for CD45 (pan leucocyte marker) and further confirmed using CD16 (PMN), mCD14 (monocytes), HLA-DR (macrophages) and CD3 (lymphocytes). Surface marker expression was measured as mean fluorescence intensity (MFI).

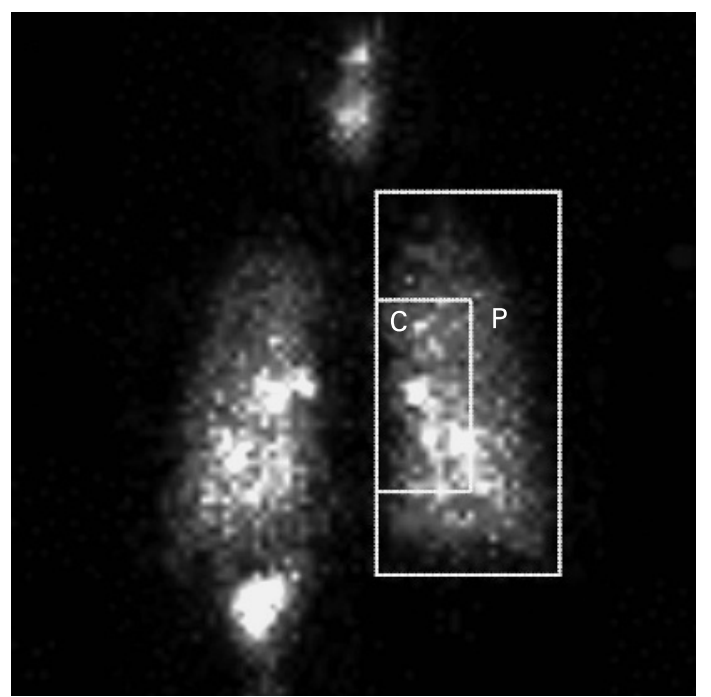

Figure 2 Posterior gamma camera image of the whole lung showing regions used for determination of the central to peripheral $(\mathrm{C} / \mathrm{P})$ ratio and to evaluate the regional deposition of inhaled particles. The larger rectangle outlines the whole right lung as defined by a xenon equilibrium scan, while the smaller rectangle defines the central region (C), comprised of a higher proportion of large airways relative to the lung periphery $(\mathrm{P})$. The very bright areas within the lung represent radioactivity concentrated in central airways. 
In vitro phagocytosis of FITC-labelled opsonised Saccharomyces cerevisiae zymosan-A bioparticles (3-4 $\mu \mathrm{m}$ physical diameter; Molecular Probes, Eugene, Oregon, USA) was assessed by measuring a shift in MFI of gated populations as an index of phagocytic activity. The number of particles engulfed per cell was estimated as the ratio of the MFI of the phagocytic cell population and the MFI of fluorescent zymosan particles. In addition to the macrophage population as a whole, phagocytosis by macrophage subpopulations (ie, mature and immature macrophages) ${ }^{14}$ were also evaluated separately.

Fluid phase cytokines (interleukin (IL) $1 \alpha$, IL1 $\beta$, IL4, IL5, IL6, IL8, IL10, IL12p, IL13, tumour necrosis factor (TNF) $\alpha$, interferon (IFN) $\gamma$, monocyte chemotactic protein (MCP)-1, macrophage inflammatory protein (MIP)- $1 \beta$, granulocyte-macrophage colony stimulating factor (GM-CSF), RANTES and eotaxin) were analysed using Luminex bead array technology (Luminex100 Total System, Luminex Corporation, Austin, Texas, USA).

\section{Statistical analysis}

Based on our previous study ${ }^{6}$ where we found mean (SD) uptake of $27(12) \%$ in a group of healthy subjects without asthma, we calculated that we would be able to detect a change of $\pm 15 \%$ in in vivo percentage uptake (primary end point) with a total sample size of 18 (power $=0.75$ for two-tailed test, $\mathrm{p}<0.05$ ). Secondary exploratory end points included particle deposition and retention measurements, total and differential cell counts, flow cytometric measurements and sputum fluidphase cytokine analysis. Specific variables were compared using two-tailed non-parametric statistics (Mann-Whitney U test and Wilcoxon sign rank test, as appropriate) and the significance of correlations was tested using Spearman's correlation. Multivariate analysis was used to assess the dependence of average particle retention on subject type (healthy vs asthmatic) and $\mathrm{C} / \mathrm{P}$ ratio of deposited activity, the latter variable included in the analysis because it has been shown previously that average particle retention is a function of deposition pattern in the lung. ${ }^{.10}$ An overall level of $p \leqslant 0.05$ was considered to be significant. All values are expressed as the mean (95\% confidence interval, CI). GraphPad Prism V.3.1 statistical software (GraphPad Software) was used for statistical analysis, except that Systat V.5.2 for MacIntosh was used for multivariate analyses.

\section{RESULTS}

\section{Sputum cell counts}

One subject with asthma was unable to produce an adequate sample and was excluded from the analysis of sputum parameters. Total and differential cell counts and other sputum parameters for normal subjects and those with asthma are presented in table 1. No significant differences were detected between the two groups for any of the sputum parameters except for the percentage of eosinophils which, although very low, was significantly higher $(p<0.001)$ in the subjects with asthma. Use of anti-inflammatory medications by asthmatic subjects may, at least in part, explain the slightly lower percentage of neutrophils in their sputum samples.

\section{In vivo uptake of inhaled sulfur colloid (SC) particles}

In vivo particle uptake by airway phagocytic cells (fig $3 \mathrm{~A}$ ) was significantly greater $(p<0.01)$ in patients with asthma and was correlated with the percentage of sputum mononuclear phagocytes in the asthma group $(r=0.85, p<0.01)$ but not in the healthy subjects (fig 3B). Deposition of inhaled particles tended

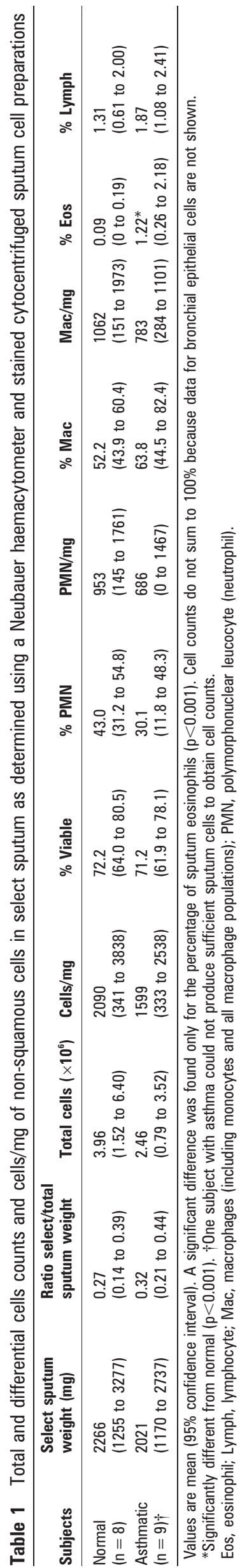



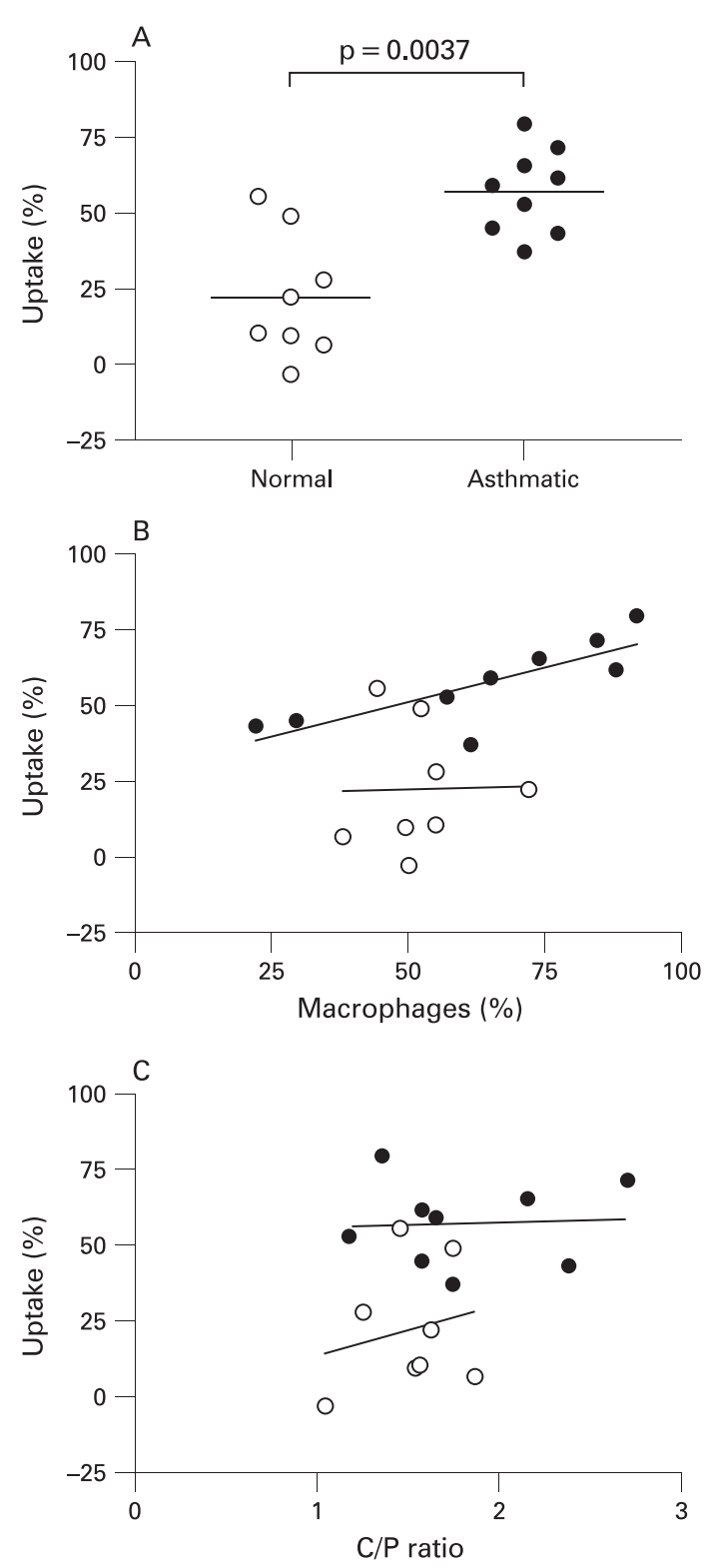

Figure 3 (A) Corrected in vivo percentage uptake of radiolabelled sulfur colloid particles was significantly greater in subjects with asthma than in healthy subjects $(57.2 \%$ (95\% Cl $46.5 \%$ to $67.9 \%)$ vs $22.3 \%(4.9 \%$ to $39.6 \%), p<0.01$ ). (B) Uptake of sulfur colloid particles was significantly correlated with the percentage of mononuclear phagocytes in induced sputum for subjects with asthma $(r=0.8500, p<0.01)$ but not for normal healthy subjects. (C) In vivo particle uptake was not a function of the site of deposition as assessed by the lack of significant correlation with the central to peripheral $(C / P)$ ratios for either normal subjects or those with asthma. Normal subjects (open circles, $n=8$ ); subjects with asthma (closed circles, $n=9$ ).

to be slightly more centralised in the airways of subjects with asthma (higher $\mathrm{C} / \mathrm{P}$ ratio); however, the mean $\mathrm{C} / \mathrm{P}$ ratio was not significantly different $(p=0.24)$ from that in healthy subjects. There was no significant correlation between the $\mathrm{C} / \mathrm{P}$ ratios and in vivo particle uptake for normal subjects or those with asthma (fig 3C).

Although some amount of radioactivity could be detected in the non-selected sputum sample portions, there was no significant difference in the amount of radioactivity between healthy subjects and those with asthma (41.7\% (95\% CI 22.0\% to $61.5 \%$ ) vs $35.0 \%$ (95\% CI $15.5 \%$ to $54.6 \%$ ) respectively, $p=0.42$ ). Much of this probably resulted from non-cellassociated free SC particles. Thus, their exclusion from uptake calculations on the selected sample may have resulted in an overestimation of uptake (up to $35-40 \%$ as an average relative amount). Separate experiments determined that DTT had no effect on the integrity of the ${ }^{99 \mathrm{~m}} \mathrm{Tc}$ label to remain affixed to the SC particles.

\section{Flow cytometry}

Flow cytometric assays were performed on only $7 / 8$ healthy subjects and $7 / 10$ subjects with asthma owing to one of the following causes: a sputum sample could not be obtained, insufficient cells for adequate flow cytometric analysis, accidental loss of cell sample or instrument malfunction.

\section{In vitro phagocytosis assays}

It is unlikely that airway deposition of the very small (if not negligible) number of inhaled radiolabelled particles affected subsequent in vitro phagocytosis of the zymosan particles.

Sputum cell populations were discriminated on the basis of light scatter properties and phagocytosis was measured as a shift in the MFI for the gated population (fig 4). In vitro phagocytic indices were enhanced in subjects with asthma for all populations, with the exception of monocytes (table 2), due to a combination of a greater proportion of cells taking up particles and an increased number of particles engulfed per cell. Within the macrophage population as a whole, the mean proportion of mature macrophages tended to be greater (albeit not significantly) in the patients with asthma (46.9\% (95\% CI $39.2 \%$ to $54.5 \%$ ) vs $38.7 \%$ ( $95 \%$ CI $18.0 \%$ to $59.4 \%$ ), $\mathrm{p}=0.32$ ). The phagocytic index (MFI) of the mature macrophage population was significantly greater than the immature macrophage population in both the asthma $(p=0.016)$ and normal groups $(p=0.032)$, and they engulfed significantly more zymosan particles per cell than the immature macrophages ( $p=0.016$, both groups).

\section{Cell surface markers}

With the exception of CD64 and CD86, we found no significant differences in the cell surface marker expression (MFI) between subjects with asthma and healthy subjects. Expression of the coreceptor molecule CD86 was significantly increased on sputum macrophages from subjects with asthma compared with healthy subjects (263 (95\% CI 126 to 400$)$ vs 141 (95\% CI 92 to 190$), p=0.05$ ) and CD64 expression was significantly lower on sputum monocytes derived from subjects with asthma (4.1 (95\% CI 1.6 to 6.7 ) vs 11.4 (95\% CI 6.2 to 16.3 ), $\mathrm{p}<0.01$ ). Although CD64 was also decreased on sputum macrophages from subjects with asthma, the difference was not significant (16.7 (95\% CI 6.5 to 27.0$)$ vs 23.7 (95\% CI 17.1 to 30.3 ), $p=0.07)$. However, exclusion of a single outlier in the asthma group would result in a significant difference from control values in the macrophage population $(p<0.01)$.

\section{Fluid phase components in sputum}

There were no statistically significant differences between normal subjects and those with asthma for any of the fluid phase cytokines examined.

\section{Whole lung particle retention and clearance}

Whole lung particle clearance was significantly more rapid in subjects with asthma $(p<0.01$, fig $5 \mathrm{~A})$, as assessed by the 

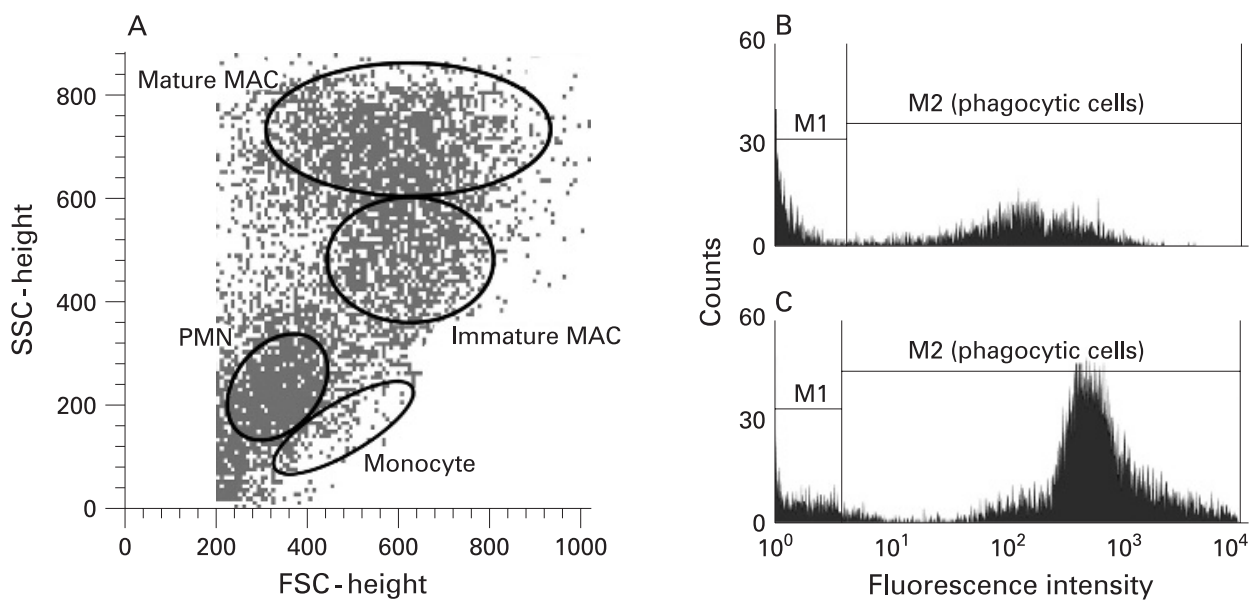

Figure 4 (A) Flow cytometry density plot showing the gating of sputum phagocytic cell populations based on light scatter properties. The right panel shows histograms of fluorescence intensity of gated macrophage populations (combined mature and immature macrophages) from (B) a normal subject and (C) a subject with asthma. Cells in the M1 region did not engulf particles while cells which engulfed particles had a higher mean fluorescence intensity (MFI) and shifted into the M2 region. Subjects with asthma were more efficient in phagocytosis of opsonised zymosan-A bioparticles (higher MFI) than healthy subjects. FSC, forward scatter; SSC, side scatter; PMN, polymorphonuclear leucocyte (neutrophil); MAC, macrophage.

average retention through the first 120 min after deposition (table 3). Patients with asthma cleared particles more rapidly (steeper slope of retention curve) during the initial 50 min after deposition than healthy subjects $(p=0.021)$. Thereafter, the slopes of the retention curves were essentially parallel. The average particle retention over the $120 \mathrm{~min}$ monitoring period correlated significantly with the $\mathrm{C} / \mathrm{P}$ ratio for subjects with asthma $(r=-0.78, p<0.01$, fig $5 B$ ) but not for healthy subjects. Whole lung particle retention was significantly less in subjects with asthma immediately following sputum induction and also at $24 \mathrm{~h}$ after deposition. The more rapid initial clearance (0-50 min) may be related to a more central deposition pattern (ie, trend towards higher $\mathrm{C} / \mathrm{P}$ ratio) in subjects with asthma. However, even for a given $\mathrm{C} / \mathrm{P}$ ratio, the subjects with asthma appear to have faster particle clearance (fig $5 \mathrm{~B}$ ), indicating that the more rapid clearance was not solely a function of more central deposition. Multivariate analysis of average retention (first $120 \mathrm{~min}$ ) as a function of both $\mathrm{C} / \mathrm{P}$ and subject type (healthy or asthmatic) showed a significant contribution from both variables $(p<0.01$ and 0.03 , respectively, $R=0.78$ for regression on both variables). Thus, the patients with mild asthma also have enhanced mucociliary clearance compared with those without asthma.

\section{DISCUSSION}

Mucociliary clearance and phagocytosis of particles by airway macrophages are generally considered to be protective mechanisms which isolate and remove potentially noxious materials (including allergens) from the airways of normal individuals. We have shown that both of these airway defence mechanisms are enhanced in subjects with mild asthma compared with healthy controls.

A significant correlation between in vivo particle uptake and the proportion of macrophages in the sputum sample (fig 3) was evident only in the subjects with asthma, indicating that enhanced particle uptake was not strictly a function of macrophage numbers or percentages. Our in vitro findings (table 2) suggest that this could be the result of a higher state of activation of airway phagocytes (both macrophages and

Table 2 Indices of phagocytosis for various phagocytic cell populations after in vitro phagocytosis of FITC-labelled opsonised zymosan-A bioparticles*

\begin{tabular}{|c|c|c|c|c|c|}
\hline & \multirow[b]{2}{*}{ PMN } & \multicolumn{4}{|c|}{ Mononuclear phagocyte populations } \\
\hline & & Monocytes & All macrophages & Immature macrophages & Mature macrophages \\
\hline \multicolumn{6}{|c|}{ Population phagocytic index (MFI) } \\
\hline Normal & 67 (42 to 91$)$ & $67(29$ to 105$)$ & 170 (111 to 229$)$ & 134 (90 to 177$)$ & 219 (147 to 292$)$ \\
\hline Asthmatic & $356(150$ to 563$)$ & $86(1$ to 171$)$ & 767 (199 to 1337$)$ & 560 (147 to 973$)$ & $1016(293$ to 1738$)$ \\
\hline p Value & 0.001 & 1.000 & 0.001 & 0.002 & 0.001 \\
\hline \multicolumn{6}{|c|}{ Phagocytic cells within a population (\%) } \\
\hline Normal & 24.8 (16.2 to 33.4$)$ & $22.8(12.2$ to 33.5$)$ & 46.4 (33.0 to 59.8$)$ & 42.8 (31.2 to 54.3$)$ & 41.5 (29.8 to 53.2$)$ \\
\hline Asthmatic & 58.4 (39.9 to 76.9$)$ & $29.5(11.3$ to 47.7$)$ & 69.6 (59.9 to 79.2$)$ & $81.5(68.5$ to 94.6$)$ & $67.0(57.7$ to 76.37$)$ \\
\hline p Value & 0.001 & 0.710 & 0.004 & 0.001 & 0.001 \\
\hline \multicolumn{6}{|c|}{ Particles/phagocytic cell } \\
\hline Normal & $1.7(1.0$ to 2.3$)$ & $1.8(1.2$ to 2.3$)$ & $2.3(1.8$ to 2.7$)$ & $1.7(1.4$ to 2.0$)$ & $2.4(1.8$ to 3.0$)$ \\
\hline Asthmatic & $3.0(1.6$ to 4.3$)$ & $1.4(0.9$ to 1.9$)$ & $6.0(2.4$ to 9.6$)$ & $3.6(1.3$ to 5.9$)$ & 7.7 (2.9 to 12.4$)$ \\
\hline $\mathrm{p}$ Value & 0.053 & 0.259 & 0.001 & 0.011 & 0.001 \\
\hline
\end{tabular}

Values are mean (95\% confidence interval). Subjects with asthma generally demonstrated enhanced phagocytosis compared with normal healthy subjects.

*Normal subjects $(n=7 / 8)$; subjects with asthma $(n=7 / 10)$; assays were not done for some subjects (see text).

All macrophages, combined immature and mature macrophage populations, excluding monocytes; MFI, mean fluorescence intensity; PMN, polymorphonuclear leucocyte (neutrophil). 

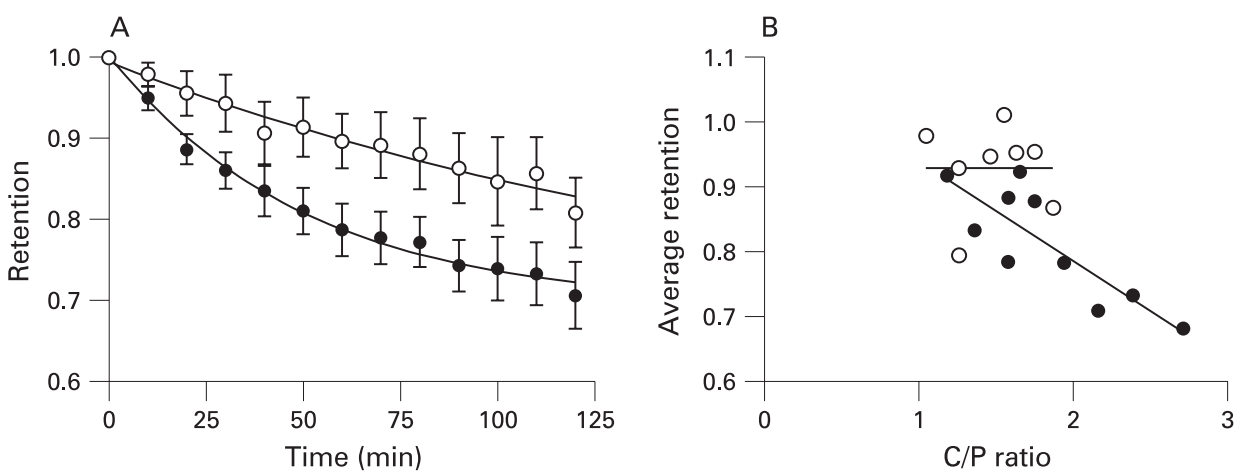

Figure 5 (A) Whole lung particle clearance proceeded more rapidly in subjects with asthma during the first 120 min after deposition than in healthy subjects, with asthmatic subjects clearing particles at a more rapid rate during the first 50 min after inhalation (see table 3). (B) The average retention during the first 120 min correlated significantly with the central to peripheral $(C / P)$ ratio for subjects with asthma $(r=-0.78, p<0.01)$ but not for normal healthy subjects. Normal subjects (open circles, $n=8$ ); subjects with asthma (closed circles, $n=10$ ).

neutrophils) in subjects with asthma. Diminished expression of CD64 on sputum macrophages and the lack of a correlation between particle uptake and surface expression of either CD64 or $\mathrm{CD} 11 \mathrm{~b}$ suggest that factors other than $\mathrm{F} c \gamma$ or complement receptor-mediated phagocytosis were responsible for the increased phagocytic activity in the subjects with asthma. For instance, scavenger receptors such as MARCO $^{15}$ or CD206 (macrophage mannose receptor) and others can also mediate phagocytosis. ${ }^{16}$ Differences in the in vivo airway surface liquid milieu of patients with asthma - such as airway mucins, ${ }^{17}$ surfactant proteins, ${ }^{18}$ phospholipids, ${ }^{19} 20$ adenosine, ${ }^{21} 22$ airway hydration or differential responses to albuterol or hypertonic saline-may also have influenced particle-macrophage interactions.

Beta-2 adrenergic agonists delivered acutely are reported to increase mucociliary clearance, ${ }^{23}$ although others found little or no effect ${ }^{24}$ relative to placebo in patients with stable asthma. Because albuterol was not administered until just before sputum induction, it could not have influenced either in vivo particle uptake or mucociliary clearance of SC particles. The extent to which regular use of albuterol by our subjects with asthma may have influenced our measurements of either particle uptake or mucociliary clearance is unclear. Routine use of $\beta_{2}$ agonists by our subjects with asthma may explain the decreased CD64 on their sputum macrophages. ${ }^{25}$ Nevertheless, it is unlikely that albuterol administered acutely contributed significantly to the observed differences in in vitro phagocytosis during the relatively short duration of sputum induction and processing.

We have considered that our results may have been subject to a sampling bias, due in part to slightly more central particle deposition in the asthma group which contributed to a more rapid initial rate of clearance (first $50 \mathrm{~min}$ ) and lower average particle retention over the $2 \mathrm{~h}$ monitoring period. However, the proportion of the lung particle burden retrieved by sputum induction was not significantly different between the two groups (table 3), and neither regional deposition nor clearance kinetics was correlated with in vivo percentage uptake of SC particles, suggesting that sampling bias did not contribute to the difference in particle uptake. The movement of particles into other airway compartments, such as bronchial epithelium or interstitial locations, tends to occur over much longer periods ${ }^{26}{ }^{27}$ than the short $2 \mathrm{~h}$ time frame of our measurements and is unlikely to have played any significant role in our measurements.

Our current in vitro findings parallel our previous findings ${ }^{5}$ and those of others ${ }^{28}$ and demonstrate enhanced phagocytic capacity of airway macrophages in subjects with mild asthma. In our previous study, in vitro phagocytic activity of sputum macrophages was diminished in subjects with more severe asthma, similar to more recent findings in children with asthma. ${ }^{29}$ In addition, as shown in this study (fig 5) and previous studies, mucociliary clearance is enhanced in mild asthma ${ }^{58}$ but clearly depressed both with increasing disease severity $^{30}$ and during acute exacerbations. ${ }^{31}$ These various studies demonstrate differences in the phagocytic functionality of sputum phagocytes, not only between healthy subjects and those with asthma but also between subgroups of patients with asthma which may be related to the severity of their disease.

Enhancement of in vivo particle uptake by airway phagocytes may play a protective role in patients with mild allergic asthma by sequestering allergens from Th2-committed mucosal dendritic cells. However, airway macrophages-like dendritic cells-also function as antigen presenting cells and have been shown to have constitutively altered functional ${ }^{52}$ and phenotypic $^{32}$ characteristics favouring a Th2 commitment and clearly play a role in the pathogenesis and maintenance of allergic airways disease. ${ }^{33}$ The constitutively increased expression of CD86 on macrophages in our cohort of patients with asthma supports the notion of enhanced antigen presenting capability and is consistent with these previous findings.

We acknowledge that a potential pitfall in this study is that we have compared numerous parameters in a relatively small number of subjects. Despite this, our conclusions regarding our stated primary end point (ie, differences in the in vivo uptake of inhaled particles) are supported by robust data and valid statistical evaluation. We note, however, that the sheer number of comparisons of secondary parameters could increase the probability of type 1 or type 2 statistical errors (false positive or false negative test) due to chance alone. In particular, statistical comparisons of those parameters having less robust differences and/or data derived from less than the full complement of subjects (eg, flow cytometric data for cell surface markers) may be more vulnerable to spurious findings.

\section{CONCLUSION}

In vivo uptake of inhaled particles and in vitro phagocytosis of opsonised bioparticles by airway macrophages are enhanced in subjects with mild allergic asthma compared with healthy subjects, as is mucociliary clearance of particles from the central airways. These findings prompt us to speculate that enhanced uptake of ambient particles and allergens coupled with 
enhanced constitutive expression of co-stimulatory molecules (ie, CD86) by Th2-biased airway mononuclear phagocytes could ostensibly lead to more efficient capture, processing and presentation of inhaled environmental allergens to Th2-committed T lymphocytes. Confirmation of this hypothesis requires additional research.

Acknowledgements: The authors thank Margaret Herbst, Martha Almond, Carole Robinette, Lynn Newlin-Clapp, Fernando Dimeo and Heather Wells (UNC-CEMALB) for their efforts in subject recruitment, technical and clinical assistance. They also acknowledge and thank Lisa Rothlein for cytokine analysis of sputum supernatant samples at the UNC-CEMALB Core Proteomics Laboratory (Dr Robert A S Roubey, Director).

Funding: This research was supported primarily through a grant from the National Institutes of Health (NHLBI-R01 HL080337) and through a cooperative agreement with the United States Environmental Protection Agency. Although the research described in this article has been funded in part by the United States Environmental Protection Agency through cooperative agreement CR83346301 with the Center for Environmental Medicine, Asthma and Lung Biology at the University of North Carolina at Chapel Hill, it has not been subjected to the Agency's required peer and policy review and therefore does not necessarily reflect the views of the Agency and no official endorsement should be inferred. Mention of trade names or commercial products does not constitute endorsement or recommendation for use.

Competing interests: None.

Ethics approval: This study was approved by the University of North Carolina Committee for the Rights of Human Subjects (Institutional Review Board).

Patient consent: Prior to participation in the study, all subjects read and signed an informed consent form after having the opportunity to ask questions regarding the purpose of the study, procedures and associated risks.

\section{REFERENCES}

1. Samet JM, Zeger SL, Dominici F, et al. The National Morbidity, Mortality, and Air Pollution Study. Part II: Morbidity and mortality from air pollution in the United States. Res Rep Health Eff Inst 2000;94:5-70.

2. Goldsmith CA, Kobzik L. Particulate air pollution and asthma: a review of epidemiological and biological studies. Rev Environ Health 1999;14:121-34.

3. Alexis NE, Lay JC, Zeman K, et al. Biological material on inhaled coarse fraction particulate matter activates airway phagocytes in vivo in healthy volunteers. J Allergy Clin Immunol 2006:117:1396-403.

4. Ormstad H. Suspended particulate matter in indoor air: adjuvants and allergen carriers. Toxicology 2000;152:53-68.

5. Alexis NE, Soukup J, Nierkens S, et al. Association between airway hyperreactivity and bronchial macrophage dysfunction in individuals with mild asthma. Am J Physiol Lung Cell Mol Physiol 2001;280:L369-75.

6. Alexis NE, Lay JC, Zeman KL, et al. In vivo particle uptake by airway macrophages in healthy volunteers. Am J Respir Cell Mol Biol 2006;34:305-13.

7. Geiser M, Cruz-Orive LM, Im Hof V, et al. Assessment of particle retention and clearance in the intrapulmonary conducting airways of hamster lungs with the fractionator. J Microsc 1990;160:75-88.

8. Alexis NE, Hu SC, Zeman K, et al. Induced sputum derives from the central airways: confirmation using a radiolabeled aerosol bolus delivery technique. Am J Respir Crit Care Med 2001;164:1964-70.

9. Lay JC, Berry CR, Kim CS, et al. Retention of insoluble particles after local intrabronchial deposition in dogs. J Appl Physiol 1995;79:1921-9.

10. Bennett WD, Brown JS, Zeman KL, et al. Targeting delivery of aerosols to different lung regions. J Aerosol Med 2002;15:179-88.

11. Alexis N, Soukup J, Ghio A, et al. Sputum phagocytes from healthy individuals are functional and activated: a flow cytometric comparison with cells in bronchoalveolar lavage and peripheral blood. Clin Immunol 2000;97:21-32.

12. Pizzichini E, Pizzichini MM, Efthimiadis $A$, et al. Measurement of inflammatory indices in induced sputum: effects of selection of sputum to minimize salivary contamination. Eur Respir J 1996;9:1174-80.

13. Spanevello A, Beghe B, Bianchi A, et al. Comparison of two methods of processing induced sputum: selected versus entire sputum. Am J Respir Crit Care Med 1998;157:665-8.

14. Lay JC, Alexis NE, Kleeberger SR, et al. Ozone enhances markers of innate immunity and antigen presentation on airway monocytes in healthy individuals. J Allergy Clin Immunol 2007;120:719-22.

15. Arredouani MS, Palecanda A, Koziel H, et al. MARCO is the major binding receptor for unopsonized particles and bacteria on human alveolar macrophages. $J$ Immunol 2005:175:6058-64.

16. Arredouani MS, Yang Z, Imrich A, et al. The macrophage scavenger receptor SR-AI/ $\mathrm{II}$ and lung defense against pneumococci and particles. Am J Respir Cell Mol Biol 2006;35:474-8.

17. Ordonez $\mathbf{C L}$, Khashayar $\mathrm{R}$, Wong $\mathrm{HH}$, et al. Mild and moderate asthma is associated with airway goblet cell hyperplasia and abnormalities in mucin gene expression. Am J Respir Crit Care Med 2001;163:517-23. 
18. Cheng G, Ueda T, Numao T, et al. Increased levels of surfactant protein A and D in bronchoalveolar lavage fluids in patients with bronchial asthma. Eur Respir $J$ 2000;16:831-5.

19. Gehr $\mathbf{P}$, Geiser $M$, Im Hof $\mathrm{V}$, et al. Surfactant and inhaled particles in the conducting airways: structural, stereological, and biophysical aspects. Microsc Res Tech 1993;26:423-36.

20. Wright SM, Hockey PM, Enhorning G, et al. Altered airway surfactant phospholipid composition and reduced lung function in asthma. J Appl Physiol 2000;89:1283-92.

21. Driver AG, Kukoly CA, Ali S, et al. Adenosine in bronchoalveolar lavage fluid in asthma. Am Rev Respir Dis 1993;148:91-7.

22. Huszar $\mathbf{E}$, Vass G, Vizi E, et al. Adenosine in exhaled breath condensate in healthy volunteers and in patients with asthma. Eur Respir J 2002;20:1393-8.

23. Bennett WD. Effect of beta-adrenergic agonists on mucociliary clearance. J Allergy Clin Immunol 2002;110:S291-7.

24. Guleria R, Singh TR, Sinha S, et al. Effect of inhalation of salbutamol, beclomethasone dipropionate and ipratropium bromide on mucociliary clearance in some patients with chronic stable bronchial asthma. Indian J Med Res 2003:117:158-63.

25. Paul-Eugene N, Dugas B, Gordon J, et al. Beta 2-adrenoceptor stimulation augments the IL-4-induced CD23 expression and release and the expression of differentiation markers (CD14, CD18) by the human monocytic cell line U937. Clin Exp Allergy 1993;23:317-25.

26. Churg A. Particle uptake by epithelial cells. In: Kreyling WG, Heyder J, eds. Particlelung interactions. New York: Marcel Dekker, 2000:401-35.

27. Kreyling WG, Scheuch G. Clearance of particles deposited in the lungs. In: Gehr P, Heyder J, eds. Particle-lung interactions. New York: Marcel Dekker, 2000:323-76.

28. Godard P, Chaintreuil J, Damon $\mathrm{M}$, et al. Functional assessment of alveolar macrophages: comparison of cells from asthmatics and normal subjects. J Allergy Clin Immunol 1982; 70:88-93.

29. Kulkarni N, Pierse N, Rushton L, et al. Carbon in airway macrophages and lung function in children. N Engl J Med 2006;355:21-30.

30. O'Riordan TG, Zwang J, Smaldone GC. Mucociliary clearance in adult asthma. Am Rev Respir Dis 1992;146:598-603.

31. Messina MS, O'Riordan TG, Smaldone GC. Changes in mucociliary clearance during acute exacerbations of asthma. Am Rev Respir Dis 1991:143:993-7.

32. Balbo P, Silvestri M, Rossi GA, et al. Differential role of CD80 and CD86 on alveolar macrophages in the presentation of allergen to T lymphocytes in asthma. Clin Exp Allergy 2001;31:625-36.

33. Eisenbarth SC, Cassel S, Bottomly K. Understanding asthma pathogenesis: linking innate and adaptive immunity. Curr Opin Pediatr 2004;16:659-66.

\section{Lung alert}

\section{ELISpot as a predictor for development of TB in children with TB contact}

$\mathrm{T}$ cell interferon $\gamma$ release assays such as the enzyme-linked immunospot (ELISpot) assay are widely used to diagnose latent tuberculosis (TB) infection. Currently, however, there are few data on the benefit of such tests in predicting active TB infection among exposed individuals. This study focuses on the prognostic value of such assays in children with recent household exposure to TB compared with the use of the tuberculin skin test (TST).

A total of 908 children were followed up for a mean duration of 1.3 years; $80 \%$ had been vaccinated with BCG and $76 \%$ had received isoniazid preventive treatment. The prevalence of higher TST results was significantly greater in those who had had BCG vaccination. Of the 381 children who had a positive ELISpot test, 11 developed active TB. In 550 children who had a positive TST, 12 developed active TB.

The authors conclude that there was a similar incidence rate of TB in contacts with positive ELISpot assay and positive TST results. However, the ELISpot assay detected a similar number of active $\mathrm{TB}$ cases from a fewer number of candidates. In addition, household contacts with positive ELISpot results had a 3-4-fold increased risk of progression to active TB compared with those with a negative assay. Unfortunately, quantification of this response did not further refine the risk of progression to active TB. The study was also limited by the fact that only three of the incident cases were confirmed culture positive. Also, as a high proportion of contacts had received isoniazid as preventive treatment, this may have confounded the incidence rate. In the future, ELISpot testing could provide a more succinct means of providing targeted preventive treatment to fewer contacts.

- Bakir M, Millington KA, Soysal A, et al. Prognostic value of a T-cell-based, interferon-gamma biomarker in children with tuberculosis contact. Ann Intern Med 2008;149:777-86.

\section{N Sharma}

Correspondence to: Dr N Sharma, FY2, Homerton University Hospital NHS Foundation Trust, London, UK; neelsharma1@ doctors.org.uk 